TEMAS DE ACTUALIDAD

Rev Chil Salud Pública 2020

Vol 24(1): 61-66

\title{
UNA APROXIMACIÓN SOCIOLÓGICA DEL EMPRESARIADO DE LA SALUD EN EL CHILE ACTUAL
}

\author{
A SOCIOLOGICAL APPROACH TO THE BUSINESS-IFICATION OF \\ THE HEALTH SECTOR IN CHILE
}

\section{RESUMEN}

Este trabajo tiene por objetivo realizar un análisis sociológico del empresariado de la salud en el Chile actual desde la perspectiva de la teoría de campos de Bourdieu. Para ello, se realizará una revisión estructural del modelo de salud en Chile, para luego reflexionar sobre el poder delegado del actor empresarial desde la estructura de clases de Goldthorpe y Wright. Finalmente, a partir de las características de este actor, se propone realizar investigaciones respecto de su papel en la esfera pública en un contexto de profundización del divorcio entre política y sociedad.

Palabras claves: empresariado de la salud, sociología, poder delegado, política, sociedad.

Daniel Manzano Méndez Sociólogo

Universidad de Playa Ancha, Máster en Análisis Político (Universidad Complutense de Madrid); Doctorante en Ciencias sociales

(Universidad de Chile) daniel.manzano@uchile.cl

Recibido el 11-11-2019; Aprobado el 27-04-2020

\section{ABSTRACT}

This work aims to perform a sociological analysis of health entrepreneurship in today's Chile from the perspective of Bourdieu's field theory. We carry out a structural review of the health model in Chile and then reflect on the delegated power of the business actor from the Goldthorpe and Wright class structure. Finally, based on the characteristics of this actor, it is proposed to research his role in the public sphere in a context of deepening the divorce between politics and society.

Keywords: health entrepeneurship, sociology, delegated power, politics, society. 
Para analizar al empresariado de la salud, se situará su posición en el marco de la teoría de campos de Bourdieu. Por tanto, este ensayo se posiciona desde la superación del debate sociológico de la falsa dicotomía entre estructura y agencia. Esto permite comprender que este actor se sitúa en un campo de relaciones de poder, en el cual se disputan distintos intereses bajo una estructura objetiva que tiene fijadas ciertas reglas del juego que lo condicionan. Sin embargo, siempre existirán agentes e instituciones que luchan constantemente por dominar el campo en disputa, abriendo la posibilidad de cambiar las reglas del campo. Por tanto, no existen actores hegemónicos ni subalternos predeterminados, ni tampoco plena libertad de movimiento de los agentes sin estructuras que condicionen la disputa de intereses.

Al adoptar esta mirada se invitará a separar el análisis en dos niveles. Por un lado, la estructura del campo de salud pública, abordando las principales características del modelo de salud neoliberal que expresarán las reglas del campo de la salud pública, redefiniendo lo que es "público" y lo "privado". En un segundo nivel se hará una caracterización e interpretación del empresariado de la salud, desde la perspectiva neoweberiana de Goldthorpe y neomarxista de Wright para analizar la estructura de clases. Ambos, al igual que Bourdieu, se sitúan en una superación del determinismo economicista.

Esta interpretación adoptará un carácter historicista. No sólo porque el fenómeno a estudiar se enmarca en un contexto político, social, económico situado, sino que también por la capacidad de agencia de los actores involucrados, permitiendo abrir la capacidad de acción sin definiciones predeterminadas.

\section{NEOLIBERALIZACIÓN DE LA SALUD}

El 11 de septiembre de 1973, se terminó por poner fin al intento de crear un sistema único de salud. Se fusiona el Servicio Médico Nacional de empleados (Sermena) y el Servicio Nacional de Salud (SNS) en el nuevo Sistema nacional de servicios de Salud (SNSS). Se crean dos tipos de aseguradoras. El seguro de salud del Estado denominado Fondo Nacional de Salud (Fonasa), el cual se constituye como sistema de reparto, y las aseguradoras privadas catalogadas como Institutos de salud previsional (Isapre), las cuales se constituyen bajo una lógica de capitalización individual.

Durante el año 1979 adicionalmente se profundiza y amplía la población beneficiaria de la mo- dalidad de libre elección (MLE), que anteriormente era de uso exclusivo para funcionarios del Sermena, terminándose de perfeccionar estas modificaciones a la MLE en 1985 con la Ley 18.469 que generó la sistematización de los aranceles de la MLE y la modalidad de atención institucional (MAI) del Fonasa. Esta modalidad es un caso de subsidio a la demanda que busca fortalecer la libre elección de los consumidores con el fin de estimular el mercado privado de la salud.

En el seguro de salud, se elimina el aporte del empleador, se aumenta el aporte a salud del trabajador, se constituye la afiliación de carácter individual al sistema Isapre y el establecimiento de incentivos estatales para aumentar la incorporación de usuarios al sistema privado. Según Ernesto Miranda "el gasto público en salud alcanza para el año 1972 un 4\% del producto geográfico bruto y para el año 1988 este llega a 2,4\%, de acuerdo con el autor esta reducción responde en parte al crecimiento de las Isapres, como entes que compiten por la cotización de los ciudadanos con la alternativa pública FONASA"1.

Durante los años 80 se instala una primera ola de privatizaciones en Chile, en donde las empresas estatales son vendidas al empresariado local. Ya en los años 90, surge una segunda ola, pero esta vez dirigida a la mercantilización de las condiciones de vida de las personas, es decir de la educación, la salud y las pensiones. En el caso de la salud, explota el boom del mercado de clínicas y consultorios privados, teniendo como principal objetivo obtener ganancia económica. A comienzos de los años 90, surgen una serie de reformas respaldadas por el Banco Mundial que buscarán profundizar la privatización de la salud y la colaboración del Estado con el empresariado. Entre ellos el proyecto Technical Assistance and Hospital Rehabilitation Project (TAHRP) y Health Sector Reform Project (HSRP), ambos con apoyo financiero de la entidad internacional.

El sistema de atención tendrá una dualidad público-privada. En 1994, el Ministerio detalla que el "sector privado se inserta en la red asistencial por la vía de la venta de servicios a los beneficiarios del sector público, según las modalidades de compra, definidas en el propio sector mediante el DFL 36 vigente -delegación de acciones-y el uso de la modalidad de libre elección del FONASA"2. Además, para garantizar esta dualidad, se impulsó un proceso de definición y separación de funciones, el cual fue apoyado a través de estudios del proyecto HSRP. Se fortaleció como entidad reguladora la superinten- 
dencia de Isapres, creada bajo la ley $\mathrm{N}^{\circ} 18.933$ en 1990, la cual buscará regular y supervisar el mercado de seguros de la salud. Además, se incentivó la regulación de los prestadores por vía de la acreditación de establecimiento, incentivos al desempeño y adscripción territorial de los beneficiarios. En ese contexto, el ministro Jiménez del primer gobierno de la Concertación de Partidos por la Democracia, en su documento "Problemas y desafíos del sistema de salud chileno" de 1991, plantea entre las políticas de salud específicas, la intención de impulsar "incentivo económicos a las Isapres para que den atención a largo plazo, entre otras medidas" ${ }^{2}$.

Los organismos internacionales comenzarán a direccionar la alianza público-privada generando ciertas recomendaciones a los sistemas de salud. La OMS, a través del Informe del año 2000 denominado "Mejorar el desempeño de los sistemas de salud", marcará un hito en la consolidación de la separación de funciones de rectoría y las funciones de prestación de servicios, es decir se comienza a respaldar la idea que, si bien la rectoría debe ser asumida por el Estado, la prestación de servicios puede ser ejecutada tanto por entidades privadas (con o sin fines de lucro) como por entidades estatales ${ }^{3}$. A su vez, las recomendaciones de la OCDE promueven que para mejorar la salud de las personas se necesitan proyectar alianzas público/privadas. ${ }^{4}$

Durante el gobierno de Ricardo Lagos, se impulsó una reforma en la cual se priorizaron algunos problemas de salud, por sobre otros, esto se efectúa según su carga enfermedad, prevalencia en la población y considerando preferencias sociales. Esto se tradujo en un conjunto de problemas de salud que cuentan con un guía que estandariza para el cuidado, se establece un sistema de garantías explícitas, exigibles al Estado: a la oportunidad, donde se establecen umbrales de atención máximos, financiamiento, se establece un mecanismo de valorización de las prestaciones y una cobertura financiera garantizada por el Estado. Posteriormente la garantía de calidad, la cual establece requisitos mínimos de calidad que deben ser cumplidos por los prestadores públicos y privados. Esta reforma incluía una dimensión sobre el aseguramiento a la salud que fue rechazada en la discusión en el congreso. Un elemento relevante, es que las garantías obligan al Estado, en caso de que no exista capacidad en el sector público de efectuar la "prestación", a que ésta sea adquirida a prestadores privados. Esto ha impactado generando una vía de compras permanentes desde Fonasa hacia las clínicas.
Con el desarrollo del modelo neoliberal, la separación entre lo público y lo privado entendido hasta la transición democrática como la separación Estado/mercado comienza a quedar desdibujada. La salud era un aspecto aspecto de la vida cotidiana que se resolvía en la esfera pública, procesado principalmente a través de los derechos sociales garantizados por el Estado y sus instituciones. No obstante, dichos aspectos constitutivos de "lo público" son privatizados, es decir se transfiere la responsabilidad de obtener salud al esfuerzo de cada individuo dependiendo de su capacidad de pago.

Según el sociólogo Sennet, surge el declive del hombre público propio de la modernidad ${ }^{5}$, es decir una crisis de la cultura pública y el surgimiento de la tiranía de la intimidad. Se ha desgastado el equilibrio de la vida privada y pública, que, según el autor, existía en el contexto incipiente del desarrollo capitalista. Por otro lado, aquellos aspectos que anteriormente eran propio de "lo privado", como por ejemplo el ámbito doméstico y familiar, comienzan a ser parte del debate público.

\section{EMPRESARIADO DE LA SALUD EN EL CHILE ACTUAL}

En este contexto surge el empresariado de la salud, el cual se ha organizado principalmente a través de los denominados holdings. Estos surgen a través de la superación del viejo paradigma productivo que se encontraba vigente en los países capitalistas industrializados, el cual "se caracterizaba por la dominación de grandes empresas organizadas jerárquicamente y la producción masiva de productos homogéneos, utilizando máquinas herramientas de propósitos únicos"6. Mientras que las nuevas "formas de la competencia entre capitales se establecen a través de un tejido de acuerdos, relaciones de cooperación, de subcontratación y de integración horizontal, definida por los constantes cambios de propiedad a partir de compraventas de empresas agrupadas en grandes holdings" ${ }^{\prime 7}$.

En Chile, a partir del informe "Análisis de la concentración en la industria de las Isapres" del Departamento de Estudios de la Superintendencia de Isapres del año 2001, se describe el mercado de la salud y su composición organizacional. Se evidencia que al año 2001, "el 54,9\% de la propiedad del mercado lo controlan cinco socios, la Cámara Chilena de la Construcción (CCHC), Aetna, la familia Fernández León, el grupo Penta y Cigna". El grupo 
Banmédica S.A, la principal fuerza del mercado, seguido por la CCHC y finalmente Aetnea. El Informe advierte que, dado su concentración en el mercado de la salud, existen riesgos de prácticas monopólicas que estos últimos tres consorcios manejaban el $64 \%$ del mercado.

En aquel Informe se comienza a describir el concepto de "integración vertical" para la operación comercial de las Isapres con los prestadores para controlar mejor los costos en salud. En algunos casos, esto "se ha traducido en adquisiciones totales o de gran parte de la propiedad de clínicas, centros médicos, laboratorios, así como el empleo directo de profesionales de la salud (médicos, enfermeras, tecnólogos médicos, matronas, etc.) ${ }^{\prime \prime}$. Algunas de ellas pertenecen a los Ilamados holdings.

A partir del desarrollo de la integración vertical descrita, esta quedará prohibida por ley para el año 2005. De esta forma las Isapres pasan a tener "por objeto exclusivo el financiamiento de las prestaciones y beneficios de salud", en ningún caso pueden implicarse en "la ejecución de dichas prestaciones y beneficios ni participar en la administración de prestadores". Sin embargo. en el año 2013, un nuevo informe denominado "Prestadores de Salud, Isapres y Holdings: ¿Relación Estrecha?" del Departamento de Estudios y Desarrollo de la Superintendencia de Salud, describe cómo "las Isapres más relevantes del mercado han incorporado el modelo de integración vertical a través de un holdings, al cual pertenecen la Isapre y los prestadores relacionados" ${ }^{\prime \prime}$. De hecho, estos holdings presentan los mismos administradores y/o directores en las aseguradoras privadas y en los prestadores privados, como por ejemplo, en el caso del Holdings Banmédica S.A, "Héctor Concha Marambio era Director de la Isapre Banmédica y Vida tres, y además Director de Clínica Santa María, Clínica Dávila, Clínica Vespucio, Clínica Bio Bio, Clínica Ciudad del Mar, Vida integra y Help" ${ }^{\prime \prime}$.

A partir del año 2014, algunos grupos empresariales venderán el holding a multinacionales extranjeras. Estos son los casos de Banmédica S.A y Cruz Blanca salud. En el año 2014, el Grupo Bupa (British United Provident Association) concreta la compra del $56 \%$ de las acciones de Cruz Blanca Salud, cambiando su razón social a BUPA Chile S.A, adquiriendo el $100 \%$ en el año 2016. Mientras que el 28 de enero de 2018 concluye la oferta pública para la compra de acciones de Empresas Banmédica por parte de UnitedHealth Group, grupo estadounidense dedicado a ofrecer distintos servicios y productos, ya sea seguros de salud, como distintos programas de cuidado. Esta compra se comienza a profundizar en los años $2000^{10}$.

Se genera lo que Habermas denomina la anonimización del capital ${ }^{11}$, ya que no se visualiza con mayor claridad el control propietario de las empresas. Sin embargo, se fortalece la presencia de tecnocracias de alta dirección.

\section{Poder delegado del holding}

Las tecnocracias de alta dirección de los holdings operarán como un poder delegado, las cuales según la sociología neoweberiana de Goldthorpe y la sociología neomarxista de Wright, constituyen una posible nueva clase social. Cabe mencionar que, en la perspectiva de ambos autores, la clase social no se define exclusivamente por la dimensión económica, sino que también incluye la dimensión cultural y, por tanto, su capacidad de constituir sus propios intereses a través de sus relaciones sociales.

Según el sociólogo Goldthorpe, el poder delegado se establece a través de la confianza, siguiendo "dos exigencias fundamentales que el empleador, o la organización empleadora debe afrontar: primero, la que se plantea cuando es preciso delegar autoridad, y segundo, la que se plantea cuando es necesario recurrir al conocimiento especializado experto"12. En ese contexto, se genera un cierto margen legítimo de autonomía y de discrecionalidad a través del compromiso moral que mantengan con la organización. Por lo tanto, este autor, junto con los niveles de renta y condiciones de empleo (seguridad económica y posibilidades de mejora), incorpora elementos de situación en el trabajo, como por ejemplo su localización en el sistema de autoridad y control que rigen en el proceso de producción. Por tanto, define la alta dirección como una "clase de servicio".

Por su parte Wright señala que estas nuevas tecnocracias evidenciaban tener características explotadoras según una dimensión cualificadora de la explotación. En ese marco, "los asalariados altamente cualificados del capitalismo están capitalistamente explotados, pues carecen de bienes de capital, a pesar de lo cual son explotadores de cualificaciones"13. Una explotación que tiene la base en la cualificación, en el control de bienes de organización, la cual según Wright se diferencia por su pretensión a la plusvalía, o por una importancia estratégica organizacional, la cual, en la misma idea de Goldthorpe, se sustenta en la lealtad y confianza. Para el autor, este capital organizacional sería una nueva 
clase dominante.

Retomando la perspectiva de Bourdieu, dentro del campo de la salud pública existe una disputa de diversos actores que representan sus propios intereses. En ese marco, otro aspecto que sería relevante abordar en futuras investigaciones acerca de estas nuevas tecnocracias es su rol en dicho campo, sobre todo a partir de la presencia de organizaciones gremiales de aseguradoras y prestadores privados liderados por la alta dirección de las empresas. Esto resulta aún más interesante si se enmarca en un contexto de democracia restringida y, por tanto, de un distanciamiento entre la sociedad y la política institucional expresada por ejemplo en el "estallido social" o revueltas de octubre del año 2019.

Desde los años 90, se ha profundizado lo que algunos cientistas políticas denominan cómo una "cartelización" de los partidos políticos hegemónicos, es decir, cómo estos se distancian de lo social y se acoplan cada vez más al Estado ${ }^{14}$. En palabras de Habermas, "se ha refeudalizado la política, ya que se ha transformado en un espectáculo dirigido en el que los líderes y los partidos pretenden, de tanto en tanto, la aclamación plebiscitaria de una población despolitizada"11.

Uno de los factores de dicha situación de mayor consenso en el ámbito de las ciencias sociales es la instauración de lo que Moulian denomina "democracia sustantiva" o bien como señala Garretón, una "democracia protegida"15. La sustantividad consiste en "garantizar la reproducción de un orden social basado en la propiedad y la ganancia privada, la limitación de la acción colectiva de los asalariados y la tutela militar en política"16.

Si se adopta la definición de Moulian, sería interesate preguntarse por el papel del empresariado de la salud en la reproducción de este orden social basado en la propiedad y la ganancia privada, sobre todo a partir de la evidencia de cómo este ha empujado la "integración vertical" para acumular más ganancias a través de los holdings, a pesar de la prohibición legal del Estado. A su vez, según el sociólogo Carlos Ruiz ${ }^{17}$, la política ha sido secuestrada bajo ciertos intereses particulares, los cuales han sido naturalizados como intereses universales. En ese contexto, sería pertinente situar a los grupos empresariales descritos en dicha disputa de intereses a través de futuras investigaciones con el fin de sociologizar el debate de lo político.

\section{REFLEXIÓN FINAL}

Este ensayo ha buscado instalar el debate acerca de la caracterización y rol del empresariado de la salud en el campo de la salud pública. Sus principales características develan el principal interés por investigar su rol en un campo en disputa. La constitución del poder delegado y las nuevas tecnocracias organizadas en distintos gremios privados, generan una interesante aproximación sociológica para preguntarse sobre sus redes sociales y su presencia como grupo de presión en la esfera pública, sobre todo en un contexto de reglas del campo favorables para dicho empresariado, como lo es la democracia sustantiva o protegida.

Frente a ello, se invita a responder las siguientes interrogantes para futuros análisis, ¿El empresariado de la salud ha ocupado un rol en el distanciamiento entre lo social y lo político? ¿Ha sido determinante en la reproducción del orden social? ¿Qué papel ha ocupado el empresariado de la salud en la sustantividad de la democracia chilena? ¿La concentración económica e integración vertical de los holdings ha influido en el descontento político social? ¿Cuál es su papel como grupo de presión en las políticas públicas del Estado?

\section{Agradecimientos:}

A mis compañeros del Doctorado en Ciencias Sociales, al Seminario "Cambios en la estructura social, actores y conflicto social en Chile" por estimular la reflexión planteada en el artículo.

\section{Declaración de conflicto de intereses:}

Sin conflicto de interés.

\section{REFERENCIA BIBLIOGRÁFICA}

1. Miranda E. Descentralización y Privatización del Sistema de Salud Chileno. Puntos Ref [Internet]. 1990;58:6-66. Available from: http://www.cep.cl/ dms/archivo_1640_1217/rev39_miranda.pdf

2. Montoya C. La salud dividida. Chile 1990-2010. Ideología, debates y decisiones acerca de la Salud: lo que dicen los documentos del periodo de los gobiernos de la Concertación. Ceibo Edic; Santiago de Chile; 2013.

3. Informe OMS. Mejorar el desempeño de los sistemas de salud; 2000.pdf.

4. Ministerio de Salud. Estudios de la OCDE sobre Salud Pública Chile hacia un futuro más sano. 2019;31. 
Available from: https://www.oecd.org/health/health-systems/Revisión-OCDE-de-Salud-Pública-Chile-Evaluación-y-recomendaciones.pdf

5. Sennett R. El declive del hombre público. 1978. Anagrama. $440 \mathrm{p}$.

6. Neffa JC. Los retos teóricos de los estudios del trabajo hacia el siglo XXI. En: CLACSO. CLACSO. Buenos Aires; 1999. p. 39-70.

7. Ruiz, C y Boccardo G. Los chilenos bajo el neoliberalismo. Clases y conflicto social. El buen Ai. Santiago de Chile; 2015.

8. Isapres Superintendencia de. Análisis de la concentración en la industria de las ISAPRES. 2001.

9. Salud Superintendencia de. Prestadores de Salud, Isapres y Holdings : ¿ Relación Estrecha ? 2013;1-6.

10. Fazio H. Mapa actual de la extrema riqueza en Chile. LOM. Santiago de Chile; 2000.

11. Habermas J. Problemas de legitimación en el campitalismo tardío. Amorrortu. Buenos aires; 1975.
12. Goldthorpe J. Sobre la clase de servicios, su formación y su futuro. Revista Zona Abierta (Madrid) 1992; No 59-60: 229-263.

13. Wright O. Reflexionando, una vez más, sobre el concepto de estructura de clases". Revista Zona Abierta (Madrid) 1992; № 59-60: 17-126.

14. Katz R, Mair P. El partido cartel: La transformación de los modelos de partidos y de la democracia de partidos. Party Politics. 1995;5-27.

15. Garretón MA. Las ciencias sociales en la trama de Chile y América Latina. Estudios sobre transformaciones sociopolíticas y movimiento social. LOM. Santiago de Chile; 2014.

16. Moulian T. Chile Anatomía de un mito. LOM. Santiago de Chile; 1997.

17. Ruiz C. De nuevo la sociedad. LOM. Santiago de Chile; 2015. 167 p. 